\title{
EFEITOS DO ULTRASSOM 3 MHz ASSOCIADO À ATIVOS LIPOLÍTICOS NA ADIPOSIDADE INFRA-ABDOMINAL: ENSAIO CLÍNICO RANDOMIZADO
}

\author{
Janaína Manhães Chartuni \\ Graduanda em Fisioterapia/ISECENSA/RJ \\ Clínica Escola Maria Auxiliadora/ISECENSA/RJ \\ janainachartuni@hotmail.com
}

Lívia Santos Sossai

Especialista em Fisioterapia Dermatofuncional/Universidade Gama Filho/RJ

Clínica Escola Maria Auxiliadora/ISECENSA/RJ

liviasossai@gmail.com

\section{Cássia Gomes Teixeira}

Especialista em Fisioterapia Dermatofuncional/Associação Pestalozzi/RJ

Clínica Escola Maria Auxiliadora/ISECENSA/RJ

cassiagt@gmail.com

\section{RESUMO}

A lipodistrofia localizada é o acúmulo regional de tecido adiposo, cujas células possuem receptores adrenérgicos $\alpha 1, \alpha 2$ e $\beta$ que controlam o processo de lipólise. O ultrassom terapêutico tem se mostrado eficaz como recurso auxiliar na redução de medidas e no combate a gordura localizada por ter sua ação efetivamente potencializada através da fonoforese. O efeito mecânico e/ou térmico do ultrassom promove modificações nas ligações intercelulares e aumento da permeabilidade da membrana celular, promovendo a difusão de substâncias através da pele. As metilxantinas (cafeína e aminofilina), além de serem bloqueadoras de $\alpha 1$, também bloqueiam a fosfodiesterase, enquanto a ioimbina bloqueia $\alpha 2$, o que contribui para a ativação da adenilatociclase e para a lipólise. O presente estudo objetivou avaliar os efeitos do ultrassom $3 \mathrm{MHZ}$ com ativos lipolíticos para redução da gordura localizada na região infra-abdominal. Em ensaio clínico randomizado experimental, duplo cego, do tipo longitudinal intervencionista, participaram 27 mulheres com faixa etária entre 18 e 30 anos, sedentárias, sem distúrbio hormonal e em uso regular de anticoncepcional. As voluntárias foram submetidas a um total de 12 sessões de fonoforese, em 3 sessões semanais, com utilização dos ativos cafeína, aminofilina e ioimbina a 2\% cada, sendo divididas em 3 grupos: Grupo US - Ultrassom 3 $\mathrm{MHz}$ contínuo com gel sem ativos lipolíticos; Grupo US+Ativos - Ultrassom $3 \mathrm{MHz}$ contínuo com gel de ativos lipolíticos, e Grupo CTRL- Ultrassom $3 \mathrm{MHz}$ contínuo desligado (grupo controle). As variáveis avaliadas foram adipometria, perimetria e ultrassonografia pré-tratamento $\left(1^{\mathrm{a}}\right.$ sessão), e pós-tratamento $\left(12^{\mathrm{a}}\right.$ sessão). Os resultados mostraram que houve redução pós-tratamento da adipometria e da perimetria infraabdominal em todos os grupos quando comparados aos valores pré-tratamento $(p<0.05)$. Não houve diferença da ultrassonografia pós-tratamento quando comparada à pré-tratamento $(\mathrm{p}>0.05)$. $\mathrm{O}$ grupo US+Ativos apresentou diferença na adipometria quando comparado ao CTRL $(p<0.05)$. O grupo US apresentou diferença na adipometria, perimetria e ultrassonografia quando comparado ao CTRL $(\mathrm{p}<0.05)$. Pode-se concluir que o ultrassom terapêutico de $3 \mathrm{MHz}$ associado a ativos lipolíticos é eficaz na redução da adiposidade infra-abdominal da mesma forma que o ultrassom terapêutico na forma isolada também foi significativamente favorável e supõe-se que o grupo controle teve perda de medidas devido ao não controle de dietas.

Palavras-chave: Terapia por ultrassom; ativos lipolíticos; fonoforese; lipólise.

\section{ABSTRACT}

Localized lipodystrophy represents a regional accumulation of adipose tissue whose cells count on $\alpha 1, \alpha 2$ and $\beta$ adrenergic receptors which control lipolytic processes. Therapeutic ultrasonography has been shown as 
an efficient auxiliary resource in the reduction of measures, as well as in the elimination of localized fat once its action can be effectively potentialized by phonoforesis. Ultrasonography mechanical and/or thermal effect promotes modifications on the intercellular linkage and an increase in the cellular membrane permeability, what leads to a better diffusion of substances through the skin. Metilxantines (such as caffeine and aminofilin), besides being $\alpha 1$ blockers, also block phosphodiesterase, while ioimbin blocks $\alpha 2$, what contributes for the activation of adenylatecyclase and for lipolysis. The present study aimed to assess the effects of $3 \mathrm{MHz}$ ultrasound associated to the use of lipolytic actives in the reduction of localized fat in the infra-abdominal region. In a double-blind, longitudinal, randomized, and experimental clinic study, 27 sedentary women with ages ranging from 18 to $30 \mathrm{y}-\mathrm{o}$ (without hormonal disturbs, and in current use of contraceptive medication) were enrolled. All voluntaries underwent a total of 12 sessions ( 3 times a week) of phonoforesis with $2 \%$ caffeine, aminofilin and ioimbin each. The sample was divided in three groups: Group $U S-3 \mathrm{MHz}$ continuous ultrasound with gel and without lipolytic actives; Group US+Actives $-3 \mathrm{MHz}$ continuous ultrasound with gel containing lipolytic actives; and Group CTRL - unplugged $3 \mathrm{MHz}$ ultrasound (control group). The variables assessed were adipometry, perimetry, and pre- $\left(1^{\text {st }}\right.$ session $)$ and post- $\left(12^{\text {th }}\right.$ session) treatment ultrasound. Our findings exhibit post-treatment reductions both in infra-abdominal adipometry and perimetry for all groups when compared to pre-treatment values $(p<0.05)$. No post-treatment ultrasonography scores were found to be different from pre-treatment values ( $p>0.05)$. Group US+Actives showed differences in adipometry when compared to Group CTRL $(p<0.05)$. Group US showed differences in adipometry, perimetry and ultrasonography when compared to Group CTRL $(p<0.05)$. According to this study, It may be concluded that therapeutic $3 \mathrm{MHz}$ ultrasound associated to lipolytic actives is as effective in the reduction of infra-abdominal adiposity as therapeutic ultrasonography alone was also significantly favorable. Control group subjects loss of weight may be due to uncontrolled changes in diet.

Key-words: therapy for ultrassom; lipolytic actives; phonoforesis; lipolysis.

\section{INTRODUÇÃO}

Atualmente, as mulheres estão mais preocupadas com a gordura que se acumula em regiões localizadas do corpo, se importando com o peso corporal geral, com a prática de exercícios, e com uma alimentação mais saudável. Segundo GUIRRO \& GUIRRO (2002), a gordura presente no interior das células do tecido adiposo existe devido ao excesso de calorias ingeridas em relação à sua utilização pelo organismo.

A Fisioterapia Dermatofuncional é uma especialidade da fisioterapia que tem por objetivo a prevenção, a promoção e a recuperação físico-funcional dos distúrbios endócrino-metabólicos, dermatológicos, circulatórios e músculo-esqueléticos; apresentando-se com um caráter assistencial mais amplo em relação ao puramente "estético". Conhecimentos relevantes da anatomia, fisiologia e patologia, garantem maior seriedade à área, direcionando a forma mais eficaz de tratamento para cada paciente, potencializando e assegurando resultados efetivos, sem causar riscos a saúde (BORGES, 2006).

A lipodistrofia (gordura) localizada é o acúmulo regional de tecido adiposo. A distribuição da gordura não é uniforme em todas as regiões do corpo, havendo maior acúmulo de tecido adiposo na região proximal dos membros e na parede abdominal, especialmente nas porções laterais (SCHUH et. al. 2010). Segundo GUIRRO \& GUIRRO (2002), o padrão de distribuição da lipólise é hereditário, e pode ser associado à atividade regional de uma enzima denominada lipase lipoprotéica, que limita o ritmo de captação dos triglicerídeos pela célula adiposa.

As células do tecido adiposo possuem receptores adrenérgicos $\alpha 1, \alpha 2$ e $\beta$. Quando os receptores $\alpha$ ou $\beta$ são ativados, ocorre a inibição ou a ativação de uma enzima chamada adenilatociclase - enzima essa existente na membrana das células adiposas, que numa cascata de eventos, ativa a lipase hormônio-sensível que promoverá a "queima" da gordura. Quando o receptor $\beta$ é ativado, ele, por sua vez, ativa a enzima adenilatociclase. Por outro lado, os receptores $\alpha 1$ e $\alpha 2$ forem ativados, eles inibirão a adenilatociclase. Sempre que houver ativação dos receptores $\beta$, haverá queima de gordura. Por outro lado, toda vez que houver ativação dos receptores $\alpha$, o organismo tenderá a acumular gordura em decorrência da inibição da adenilatociclase (GUYTON, 1997; WAJCHENBERG, 2000; FONSECA-ALANIZ et. al. 2006). 
O ultrassom (US) é uma modalidade de penetração profunda, capaz de produzir alterações nos tecidos, por mecanismos térmicos e não-térmicos. Dependendo da freqüência das ondas, o ultrassom é utilizado para o diagnóstico por imagem, cura terapêutica de tecidos ou destruição de tecidos (STARKEY, 2001). O US terapêutico vem sendo empregado há mais de 40 anos no tratamento de diversas patologias e uma quantidade considerável de trabalhos laboratoriais têm sido realizados com o objetivo de esclarecer seus efeitos sobre os tecidos (KOEKE, 2003).

Os efeitos térmicos dentro dos tecidos são resultantes diretos da elevação da temperatura do tecido, provocada pelo ultrassom, variando de acordo com o coeficiente de absorção e a espessura do meio absorvedor (ERVALHO, 2005). A quantidade de absorção depende da natureza do tecido, seu grau de vascularização e da freqüência do ultrassom. Tecidos com elevado conteúdo protéico absorvem mais rapidamente que os com maior conteúdo de gordura, e quanto maior a freqüência, maior a absorção (BORGES, 2006).

GARCIA (2000) destaca, entre os efeitos não-térmicos do ultrassom, a micro-massagem, o aumento da permeabilidade celular, variação do diâmetro arteriolar e cavitação. À micro-massagem atribuem-se às oscilações provocadas pelo feixe ultrassônico que atravessa os tecidos. A movimentação desses provoca um aumento na circulação dos fluídos intra e extracelulares, facilitando a retirada de catabólitos e a oferta de nutrientes. A micro-massagem, devido ao efeito mecânico (vibrações sônicas) que o US provoca, gera calor por fricção.

A cavitação ocorre em toda aplicação de US, onde, a oscilação molecular que ocorre de maneira cíclica, promove a formação de bolhas de ar/gás. A partir disso, podem ser classificadas em estável ou instável. A cavitação estável e é aquela na qual as bolhas vibram nas ondas de pressão do US, aumentando e diminuindo de tamanho sem se romper. Esse efeito é considerado normal e desejável, pois provê efeito terapêutico. A cavitação instável ocorre quando o volume da bolha se altera bruscamente e então a bolha colapsa (implode), causando mudanças de temperatura podendo resultar em dano tecidual ou não (KOEKE,2003; GUIRRO \& GUIRRO, 2002; AGNE, 2005; JESUS et. al. 2006). Estudos mostrados por BORGES (2006), no que se refere à atuação das ondas ultrassônicas no tecido adiposo, mostram que devemos considerar o fato de que na gordura o coeficiente de absorção é baixo, decorrente da homogeneidade do tecido, absorvendo pouca energia ultrassônica, sendo pouco provável a sua ação lipolítica.

A fonoforese é o termo que descreve a introdução de substâncias medicamentoso-cosméticas através da pele, mediante a energia ultrassônica (CAGNIE \& VINCK, 2003; KOEKE, 2003; SANTOS \& XIMENES, 2010; BORGES, 2006). Essa é a técnica mais utilizada na terapêutica Dermatofuncional, empregando produtos cosméticos principalmente com ação lipolítica e estimulante da circulação. Dentre os ativos lipolíticos mais utilizados estão: a cafeína; a centella asiática; a cavalinha; a ioimbina; e a aminofilina, dentre outras. Suas funções principais são: o incremento circulatório; a ativação da lípase; a inibição e/ou ativação de alguns receptores de membrana; e a potencialização da termogênese (GUYTON, 1997; WAJCHENBERG, 2000; GUIRRO \& GUIRRO, 2002; BORGES, 2006; FONSECA-ALANIZ et. al, 2006; JESUS et. al, 2006).

As metilxantinas (cafeína e aminofilina), além de serem bloqueadoras de $\alpha 1$, também bloqueiam a fosfodiesterase. Dessa forma, o AMPc (monofosfato de adenosina cíclico) se mantém ativo, favorecendo a queima de gordura. Quanto à ioimbina, sua ação envolve o bloqueio de $\alpha 2$, o que contribui para a ativação da adenilatociclase e, como conseqüência, também para a queima de gordura (GUYTON, 1997; WAJCHENBERG, 2000; FONSECA-ALANIZ et. al, 2006).

Segundo SANTOS \& XIMENES (2010), através da adipometria podemos avaliar a dobra cutânea infra-abdominal no sentido horizontal ou vertical; a referência anatômica é a cicatriz umbilical e o procedimento deve ser realizado conforme o seguinte protocolo: dobra lateralmente três centímetros de distância da cicatriz umbilical e um centímetro abaixo do centro da cicatriz umbilical. Já a perimetria, através da utilização da fita métrica, permite a obtenção de uma medida do perímetro sem que haja compressão da pele (MARINS \& GIANNICHI, 2003). O ponto anatômico de referência para a região infra-abdominal é a cicatriz umbilical, com a fita posicionada em um plano horizontal e também com um centímetro abaixo desta (GUIRRO e GUIRRO, 2004; MARINS \& GIANNICHI, 2003). Conforme apregoado por RIBEIRO FILHO (2006), apesar de requerer equipamento e pessoal especializados à semelhança de outros, a ultrassonografia diagnóstica é um método significativamente menos dispendioso que a tomografia computadorizada, tratando-se de um método inócuo, de fácil e rápida execução e com boa especificidade e reprodutibilidade para estudo da adiposidade subcutânea e, principalmente para a adiposidade visceral. 
Na busca de um padrão estético social, é notório o aumento de indivíduos que utilizam essa e outras terapias, a fim de minimizar as disfunções estéticas decorrentes do acúmulo de gorduras localizadas, assim como também do número de procedimentos oferecidos no mercado prometendo resultados milagrosos (BORGES, 2006; SANTOS \& XIMENES, 2010). Diante dessas evidências, torna-se oportuno o estudo experimental com o objetivo de verificar os efeitos da utilização do ultrassom (3MHz), no modo contínuo, através da fonoforese, com ativos lipolíticos na redução da gordura localizada infra-abdominal e, dessa forma, contribuir para o enriquecimento e credibilidade dessa terapêutica na Fisioterapia Dermatofuncional, proporcionando acréscimo de opções para diversos tipos de tratamento, sejam eles curativos, corretivos, preventivos, ou, puramente estéticos.

\section{METODOLOGIA}

Este trabalho foi aprovado pelo comitê de ética dos Institutos Superiores do ISECENSA, através do número 0033.0.413.000-09 e todas as voluntárias assinaram o termo de consentimento de acordo com a resolução 196/96, sendo classificado como uma pesquisa experimental, ensaio clínico controlado randomizado, duplo cego.

A população desse estudo foi formada por uma amostra de 27 mulheres voluntárias da cidade de Campos dos Goytacazes (RJ), saudáveis, sedentárias, com idade entre 18 e 30 anos, todas sem distúrbios hormonais e em corrente uso de medicamentos contraceptivos. Esses dados foram considerados como critérios de inclusão. Os critérios de exclusão considerados neste estudo foram: a presença de marca-passo e de próteses metálicas na região a ser tratada; infecções; tumores; gravidez; diabetes; e/ou quadros hipertensivos não-compensados e o índice de massa corpóreo.

Neste estudo é importante destacar que as avaliações das voluntárias foram realizadas pelo mesmo avaliador, na mesma hora e no mesmo dia com o mesmo equipamento no Hospital Escola Álvaro Alvim. Para a avaliação da espessura do tecido adiposo infra-abdominal bilateral, foi utilizado o Ultrassom Diagnóstico da marca INVISOR da Phillips, cujo transdutor de alta freqüência é de $12 \mathrm{MHZ}$, através do seguinte protocolo: o transdutor foi posicionado transversalmente a $1 \mathrm{~cm}$ da cicatriz umbilical, no sentido ântero-posterior na posição adjacente a linha mediana, sem exercer nenhuma pressão sobre o abdome, a fim de não subestimar a aferição. Considera-se a espessura subcutânea como a medida entre a pele e a face externa da fáscia dos músculos reto-abdominais, quantificada em centímetros (DINIZ et. al, 2009).

Foi utilizado também o adipômetro CESCORF MITUTOYO ${ }^{\circledR}$, que tem a função de mensurar a gordura em milímetros, ressaltando-se que, nessa medição, também há a mensuração do tecido epitelial. Seguiu-se o protocolo de MARINS \& GIANNICHI (2003), o qual indica que, para validar tal avaliação, devemos medir três vezes o mesmo local referente à prega cutânea (infra-abdominal) e dessas três medidas retirar uma média.

Cabe ressaltar que tanto o US diagnóstico quanto o uso do adipômetro, estavam voltados para mensuração bilateral da região infra-abdominal.

A fim de quantificarmos a circunferência infra-abdominal, utilizamos a fita métrica como determina o protocolo de MARINS \& GIANNICHI (2003): "dois dedos abaixo da cicatriz umbilical". Tais procedimentos foram realizados no início do tratamento e no final.

Nos tratamentos, utilizamos o ultrassom terapêutico de $3 \mathrm{MHz}$ (sonopulse compact da IBRAMED ${ }^{\circledR}$ ), com a finalidade de realizar procedimentos de fonoforese com ativos lipolíticos. Nesse estudo, os ativos lipolíticos utilizados foram cafeína, aminofilina e a ioimbina a $2 \%$ cada, manipulados em farmácia de manipulação especializada, em formulação gel.

O procedimento de coleta de dados foi realizado em 12 sessões, três vezes por semana, durante 30 dias. Os indivíduos foram divididos aleatoriamente em três grupos: Grupo US - 12 SESSÕES: Ultrassom 3 MHz modo contínuo com gel sem ativos lipolíticos; Grupo US + ATIVOS - 12 SESSÕES: Ultrassom $3 \mathrm{MHz}$ modo contínuo com gel de ativos lipolíticos (cafeína, aminofilina e ioimbina a 2\% cada); e Grupo CTRL - 12 SESSÕES: Ultra -som $3 \mathrm{MHz}$ modo contínuo desligado.

O estudo foi composto das seguintes etapas:

$1^{\text {a }}$ Etapa: Avaliação fisioterapêutica e preenchimento de um questionário informando dados pessoais e respondendo às perguntas relevantes a este experimento. Logo em seguida, as voluntárias forma submetidas a uma avaliação antropométrica que se consistiu das seguintes medições: massa corporal total (peso); 
estatura (altura); dobra cutânea; e perimetria da região infra-abdominal. Também foi realizada, nessa $1^{a}$ etapa, a ultrassonografia diagnóstica da região infra-abdominal.

$2^{\mathrm{a}}$ Etapa: As voluntárias foram designadas randomicamente para um dos três grupos: Grupo US (n=10), Grupo US + ATIVOS (n=9) ou Grupo CTRL (n=8) (grupo controle).

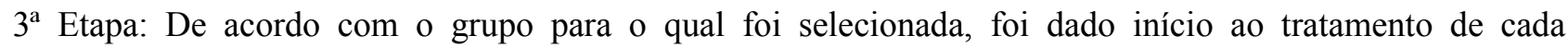
voluntária - tratamento esse que se consistiu de três sessões semanais de aplicação de ultrassom na região infra-abdominal (de acordo com o grupo sorteado). As voluntárias foram posicionadas em decúbito dorsal sobre uma maca, com os olhos vendados e região abdominal desnuda. Foi realizada a limpeza da área com álcool a 70\% e algodão, a fim de diminuir a impedância tecidual, e posterior marcação da área a ser tratada, com lápis dermográfico. Em seguida, a voluntária recebeu o tratamento proposto, com uma duração de 30 minutos no máximo.

As voluntárias foram avaliadas freqüentemente ao longo do tratamento da seguinte forma: perimetria pré-tratamento (no $1^{\mathrm{o}}$ dia) e pós-tratamento (na $12^{\mathrm{a}}$ sessão); ultrassonografia diagnóstica pré-tratamento (um dia antes da $1^{\mathrm{a}}$ sessão) e pós-tratamento (um dia após a $12^{\mathrm{a}}$ sessão); adipometria pré-tratamento (no $1^{\mathrm{o}}$ dia) e pós-tratamento (na $12^{\mathrm{a}}$ sessão).

$\mathrm{O}$ trabalho se deu em manusear o transdutor do ultrassom em movimentos circulares, lentos e uniformes, sobre a região infra-abdominal - o que, segundo BORGES (2006), proporciona melhores distribuição e absorção das ondas ultra-sônicas pelo tecido.

Para o cálculo do tempo de aplicação do ultrassom terapêutico, foi utilizada a fórmula Tempo = Área/ERA, de acordo com o apregoado por BORGES (2006). A área de tratamento escolhida foi de $10 \mathrm{~cm}^{2}$ na região infra-abdominal. Essa área foi estipulada como o dobro da área do cabeçote do ultrassom diagnóstico $\left(5 \mathrm{~cm}^{2}\right)$. Tratando-se uma área maior que a do cabeçote do ultrassom diagnóstico, buscou-se minimizar os erros na mensuração pelo posicionamento do cabeçote na área tratada, pós-tratamento. Dessa forma, se houvesse alguma alteração no posicionamento do cabeçote do ultrassom diagnóstico, ele ainda estaria dentro da área de tratamento. Respeitou-se, também, o limite de $2 \mathrm{~cm}$ abaixo da cicatriz umbilical com o objetivo de acompanhar a área de mensuração de camada adiposa realizada pelo ultrassom diagnóstico, assim como a referência de mensuração da perimetria. O tempo de aplicação do ultrassom terapêutico foi de três minutos em cada lado, totalizando um tempo de aplicação de 6 minutos.

Para a mensuração da intensidade terapêutica, considerou-se a dose ideal que deveria chegar ao tecido adiposo em estudo, levando-se em consideração a atenuação das ondas sonoras nos tecidos superficiais ao tecido-alvo (pele e tecido subcutâneo). De acordo com AGNE (2005) e BORGES (2006), recomendam-se doses abaixo de $1 \mathrm{~W} / \mathrm{cm}^{2}$ utilizando o ultrassom na forma contínua. Como não se trata de lesão tecidual, consideramos o estado tecidual em fase crônica e a intensidade requerida de $0,8 \mathrm{~W} / \mathrm{cm}^{2}$. Relacionando a intensidade requerida de $0,8 \mathrm{~W} / \mathrm{cm}^{2} \mathrm{com}$ a profundidade do tecido, no caso desse estudo, a espessura do tecido adiposo de cada voluntária dado através do ultrassom diagnóstico, chegou-se à intensidade a ser utilizada em cada voluntária - intensidade essa que variou de $1,12 \mathrm{~W} / \mathrm{cm}^{2}$ a $2 \mathrm{~W} / \mathrm{cm}^{2}$.

Para garantir a confiabilidade das doses, como sugeriu HERCOX et. al, (1994), multiplicamos o valor da ERA por valores relacionados ao estado tecidual em fase crônica, utilizando a fórmula Tempo $=$ Área/ERA x 1, alcançando os mesmo valores obtidos anteriormente: 3 minutos em cada lado, totalizando um tempo de aplicação de 6 minutos.

Foram consideradas variáveis dependentes do estudo: a adipometria $(\mathrm{mm})$; a perimetria $(\mathrm{cm})$; e a ultrassonografia diagnóstica $(\mathrm{cm})$. Como variáveis independentes, consideramos: a sonidoterapia e os ativos lipolíticos: a cafeína, a aminofilina e a ioimbina.

Todos os dados foram normalizados pelo momento pré-tratamento - o que garantiria o conhecimento da variação percentual das variáveis analisadas. Para a análise estatística, foi utilizado o teste $t$ pareado. $\mathrm{O}$ nível de significância adotado foi de $5 \%(\alpha=0,05)$.

\section{RESULTADOS}

O presente estudo contou com uma amostra de 27 mulheres sedentárias com: idades de $23 \pm 1,2$ anos; massa corpórea total de $62,5 \pm 1,9 \mathrm{Kg}$; estatura de $162,6 \pm 1,6 \mathrm{~cm}$; IMC de $23,6 \pm 0,8 \mathrm{Kg} / \mathrm{m}^{2}$; espessura da camada adiposa de $2,8 \pm 0,2 \mathrm{~cm}$; prega cutânea de $33,6 \pm 4,8 \mathrm{~mm}$; e circunferência infra-abdominal de 
$89,4 \pm 1,9 \mathrm{~cm}$, conforme demonstrado na Tabela 1. De acordo com os dados estatísticos, não foram observadas diferenças estatisticamente significativas entre os grupos, garantindo a presença de uma amostra homogênea (Tabela 1).

Tabela 1 -Características clínicas e antropométricas dos grupos investigados (US+Ativos, US e CTRL).

\begin{tabular}{lllll}
\hline & US+Ativos & US & CTRL & $p$-valor \\
\hline Idade (anos) & $24.0 \pm 1.2$ & $23.0 \pm 1.3$ & $22.0 \pm 1.2$ & 0.538 \\
Peso $(\mathrm{Kg})$ & $61.8 \pm 2.3$ & $60.0 \pm 1.7$ & $65.7 \pm 1.8$ & 0.139 \\
Altura $(\mathrm{cm})$ & $164.2 \pm 1.2$ & $160.7 \pm 1.9$ & $163.1 \pm 1.6$ & 0.295 \\
IMC $\left(\mathrm{Kg} / \mathrm{m}^{2}\right)$ & $22.9 \pm 0.8$ & $23.3 \pm 0.8$ & $24.7 \pm 0.8$ & 0,256 \\
US diagnóstico $(\mathrm{cm})$ & $2.7 \pm 0.2$ & $3.1 \pm 0.2$ & $2.6 \pm 0.1$ & 0,206 \\
Adipometria $(\mathrm{mm})$ & $31.6 \pm 1.2$ & $34.3 \pm 1.5$ & $35.1 \pm 2.1$ & 0,091 \\
Perimetria $(\mathrm{cm})$ & $89.1 \pm 2.1$ & $88.0 \pm 1.5$ & $91.0 \pm 2.2$ & 0,551 \\
\hline
\end{tabular}

Valores expressos em media \pm erro-padrão da média das 27 pacientes no momento Pré (US+Ativos: 9 pacientes; US: 10 pacientes; CTRL: 8 pacientes). Não foram observadas diferenças estatisticamente significativas entre os grupos $(\mathrm{p}>0,05)$

A Tabela 2 apresenta os resultados da adipometria, da perimetria e do ultra som diagnóstico nos três grupos analisados (US+Ativos, US e CTRL), nos momentos pré e pós-tratamento. Em relação ao pré, foi observada diminuição da adipometria no pós-tratamento para os três grupos, representando uma redução da prega cutânea infra-abdominal bilateral em todos os grupos no momento pós-tratamento, quando comparado ao momento pré-tratamento $(\mathrm{p}<0.05)$.

Tabela 2 - Resultados referentes às variáveis de análise dos grupos investigados (US+Ativos, US e CTRL) Pré e Pós-tratamento

\begin{tabular}{llll}
\hline & US+Ativos & US & CTRL \\
\hline Adipometria $(\mathrm{mm})$ & & & \\
Pré & $31.6 \pm 1.2$ & $34.3 \pm 1.5$ & $35.1 \pm 2.1$ \\
Pós & $25.2 \pm 6.1^{*}$ & $27.6 \pm 1.1^{*}$ & $29.4 \pm 1.5^{*}$ \\
$(\Delta \%)$ & $-21.2 \pm 2.5^{\dagger}$ & $-18.4 \pm 2.1^{\dagger}$ & $-14.4 \pm 2.6$ \\
Perimetria $(\mathrm{cm})$ & & & \\
Pré & $89.1 \pm 2.1$ & $88.0 \pm 1.5$ & $91.0 \pm 2.2$ \\
Pós & $85.9 \pm 2.2^{*}$ & $84.1 \pm 1.5^{*}$ & $88.6 \pm 1.7^{*}$ \\
$(\Delta \%)$ & $-3.6 \pm 0.4$ & $-4.4 \pm 0.7^{\dagger}$ & $-2.5 \pm 0.8$ \\
Ultrassonografia $(\mathrm{cm})$ & & & \\
Pré & $2.7 \pm 0.1$ & $3.1 \pm 0.2$ & $2.7 \pm 0.1$ \\
Pós & $2.7 \pm 0.2$ & $2.8 \pm 0.2^{*}$ & $2.8 \pm 0.1$ \\
$(\Delta \%)$ & $0.3 \pm 3.6$ & $-6.4 \pm 1.8 \dagger$ & $5.5 \pm 3.6$ \\
\hline
\end{tabular}

Valores expressos em media \pm erro-padrão da média das 27 pacientes.

US+Ativos: 9 pacientes; US: 10 pacientes; CTRL: 8 pacientes.

* Diferenças estatisticamente significativas em relação ao Pré $(\mathrm{p}<0,05)$

$\dagger$ Diferenças estatisticamente significativas em relação ao CTRL $(\mathrm{p}<0,05)$

Entretanto, comparando-se as variações percentuais entre os grupos, observou-se que os grupos US+Ativos e US apresentaram maior variação quando comparados ao grupo CTRL $(p<0.05)$. Não foram observadas diferenças estatísticas entre os grupos US vs US+Ativos ( $>0.05$ ) (Fig. 1). 


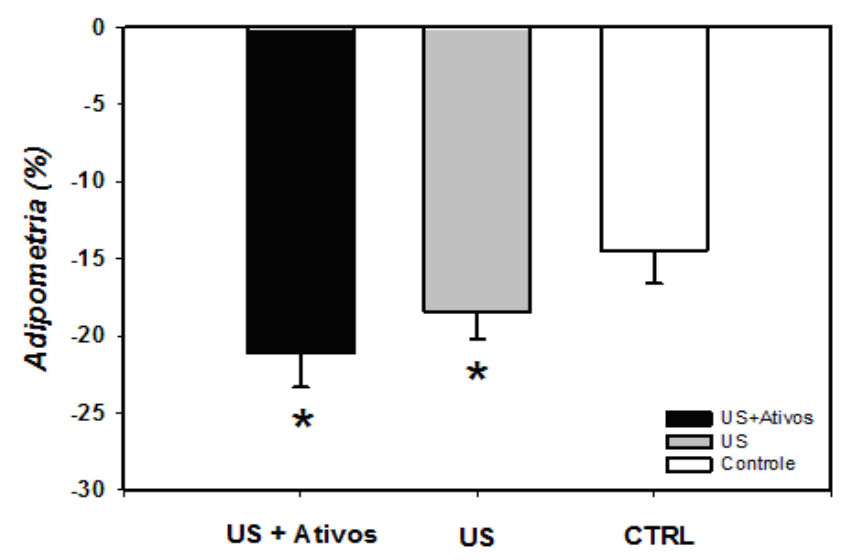

Figura 1 - Análise da variação percentual da adipometria da região infra-abdominal bilateral. (*) Grupos US+Ativos e US tiveram maior variação percentual quando comparado ao grupo CTRL $(\mathrm{p}<0.05)$.

(*) Diferença estatisticamente significativa em relação ao CTRL

No que se refere à perimetria $(\mathrm{cm})$, houve redução da circunferência infra-abdominal em todos os grupos no momento pós-tratamento, quando comparado ao momento pré-tratamento $(\mathrm{p}<0.05)$, apresentando o mesmo comportamento que a adipometria. Comparando-se as variações percentuais entre os grupos, o grupo US teve maior variação quando comparado ao grupo CTRL $(\mathrm{p}<0.05)$. Não foram observadas diferenças entre os grupos US+Ativos vs CTRL e US vs US+Ativos (p>0.05) (Fig. 2).

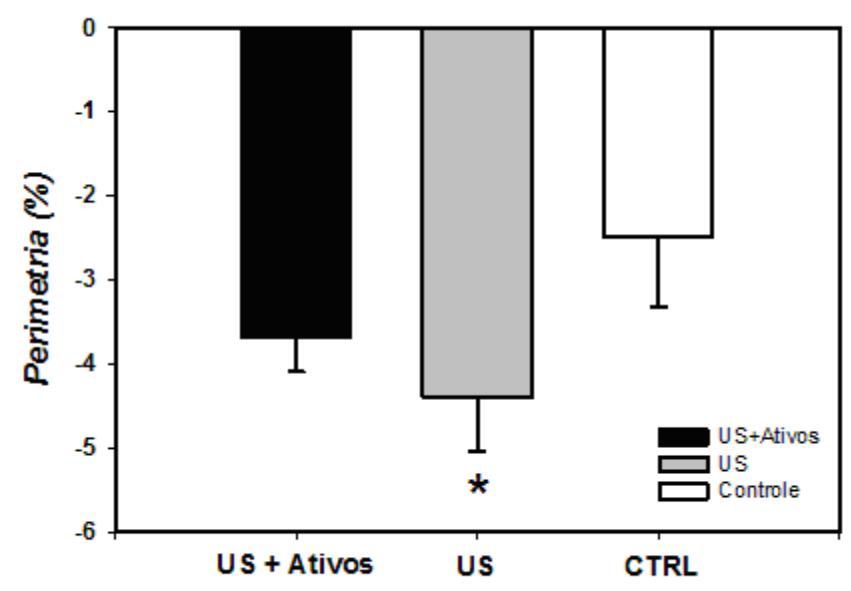

Figura 2 - Análise da variação percentual entre os grupos, no que se refere a perimetria da região infraabdominal bilateral. (*) Diferença estatisticamente significativa em relação ao grupo CTRL ( $<<0.05)$.

Através da análise da avaliação da espessura da camada adiposa pela ultrassonografia diagnóstica (Ultrassom), observou-se que, após o tratamento, somente o grupo US obteve redução significativa em relação ao pré $(\mathrm{p}<0.05)$. Comparando-se as variações percentuais entre os grupos, o grupo US apresentou maior variação quando comparado ao grupo controle CTRL $(\mathrm{p}<0.05)$. Não foram observadas diferenças entre os grupos US+Ativos vs CTRL e US vs US+Ativos ( $>0.05$ ), como demonstrado na Fig. 3. 


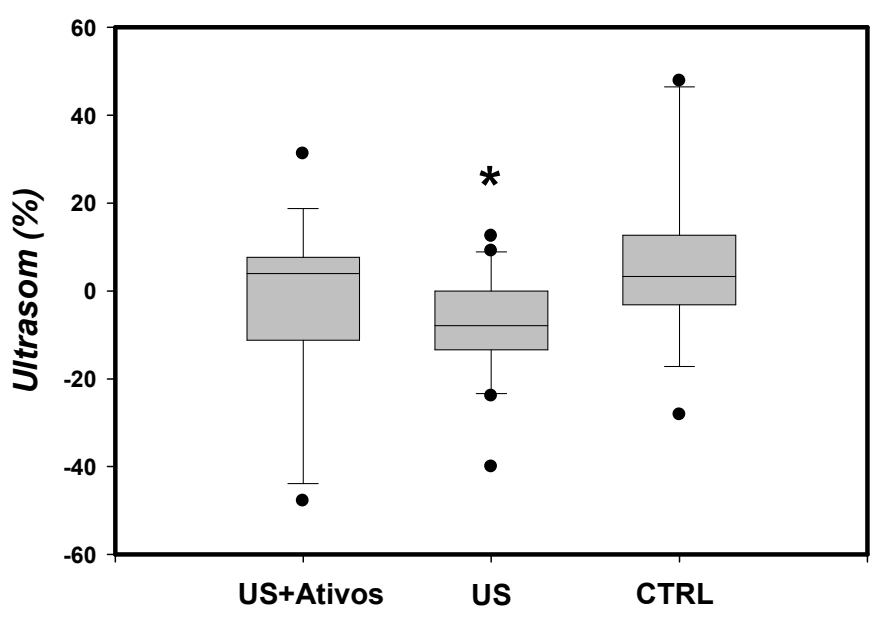

Figura 3 - Análise da variação percentual entre os grupos, no que se refere a ultrassonografia diagnóstica da região infra-abdominal bilateral. *Diferença estatisticamente significativa em relação ao grupo CTRL $(\mathrm{p}<0.05)$.

\section{DISCUSSÃO}

O presente estudo procurou verificar os efeitos da utilização do ultrassom $(3 \mathrm{MHz})$, no modo contínuo, através da fonoforese com ativos lipolíticos na redução da gordura localizada infra-abdominal e, dessa forma, contribuir para o enriquecimento e credibilidade dessa terapêutica na Fisioterapia Dermatofuncional.

De acordo com os resultados obtidos e apresentados nesse estudo, verificou-se que, em relação ao pré, foi observada diminuição da adipometria no pós-tratamento nos três grupos, representando uma redução da prega cutânea infra-abdominal bilateral em todos os grupos no momento pós-tratamento, quando comparado ao momento pré-tratamento $(\mathrm{p}<0.05)$. Entretanto, comparando-se as variações percentuais entre os grupos, observou-se que os grupos US+Ativos e US apresentaram maior variação quando comparados ao CTRL ( $\mathrm{p}<0.05)$. Não foram observadas diferenças estatísticas entre os grupos US vs US+Ativos ( $\mathrm{p}>0.05)$.

Os resultados da adipometria apresentados pelo grupo US+Ativos corroboram com os apresentados por diversos autores. Segundo Campos, et. al, (2004), o UST tem sido usado, extensivamente, nas últimas décadas como promotor de permeação cutânea de fármacos, sendo o seu uso facilitador da absorção cutânea. Segundo GUIRRO \& GUIRRO (2002), AGNE (2005) e BORGES (2006), freqüências maiores tendem a prover maior índice de transmissão, portanto, a utilização do UST de $3 \mathrm{MHz}$, comumente utilizado na Dermatofuncional, tende a prover maior qualidade quanto a permeação do produto através da pele, quando comparada ao UST de $1 \mathrm{MHz}$. Na prática clínica, recomenda-se que o UST contínuo seja utilizado em intensidades até $2 \mathrm{~W} / \mathrm{cm}^{2}$, evitando risco de lesão nas estruturas superficiais (AGNE, 2005; BORGES, 2006), Tais fatos vão ao encontro do protocolo utilizado no presente estudo, o qual utilizou US com freqüência de 3 MHz e intensidade máxima de $2 \mathrm{~W} / \mathrm{cm}^{2}$, e tendem a justificar os resultados.

Alguns autores (GUIRRO \& GUIRRO (2002), GILMAN (2003), BORGES (2006) e JESUS et. al, (2006)) defendem a atuação da fonoforese e pontuam como vantagem o fato de proporcionar ao tecido alvo o somatório dos efeitos do UST associado aos da substancia terapêutica introduzida. Para eles, a fonoforese é potencializada pela atuação do UST no aumento da permeabilidade da membrana celular, sendo capaz de promover a difusão do produto através da pele. Para TOGNI (2006) como algumas drogas são absorvidas pela pele muito lentamente, a vibração sonora de alta freqüência pode acelerar esse processo.

A utilização do UST como promotor da permeação cutânea já é uma realidade (JESUS et al, 2006). No entanto, muitos autores ainda discutem sobre qual seria a principal circunstância que envolveria a deposição da droga no tecido.

Alguns autores aconselham a utilização do pulso contínuo da onda ultra-sônica nas aplicações de fonoforese (GUIRRO e GUIRRO, 2002; BORGES et. al, 2002; BORGES, 2006). Outros, associam os efeitos mecânicos não-térmicos a uma melhor resposta tecidual em relação a permeação cutânea (LOW \& REED, 2001; STARKEY, 2001; CAGNIE \& VINCK, 2003). No entanto, JESUS et. al, (2006), em um vasto estudo sobre fonoforese e permeação cutânea, concluem que o UST, seja devido aos seus efeitos térmicos ou 
não-térmicos, favorece a permeação cutânea de produtos farmacológicos e cosmecêuticos, agindo como promotor. Contudo, parece que os efeitos térmicos têm melhores resultados quando se trata de romper o estrato córneo, pois há uma maior formação de cavitação instável, rompendo mais facilmente os tecidos. Somando a isso, os efeitos provocados pelo aquecimento da área tratada podem aumentar a absorção da medicação nos tecidos; o que vai ao encontro do presente estudo, o qual utilizou o UST no modo contínuo. Dessa forma, de acordo com o exposto pelos autores anteriormente citados, torna-se evidente a necessidade de novas pesquisas, comparando os modos contínuo e pulsado.

De acordo com KOEKE (2003) E TOGNI (2006), o responsável pela permeação cutânea seria o fenômeno de cavitação, que resulta na formação de microbolhas gasosas na camada externa da pele, que podem romper-se violentamente, aumentando a sua permeabilidade e, possivelmente permitir a passagem da droga. TOGNI (2006) ainda defende os efeitos tanto do UST pulsado quanto contínuo na facilitação da permeação cutânea. Para ele, paralelamente, o ultrassom provoca um aquecimento, o qual é capaz de aumentar a energia cinética das moléculas do fármaco e da membrana celular, dilatando os pontos de entrada dos folículos pilosos e glândulas sudoríparas e aumentando a circulação da área irradiada. Já as características mecânicas não-térmicas do UST podem aumentar a difusão de fármacos pela oscilação em alta velocidade das células, além de proporcionar mudanças no potencial de repouso da membrana celular e ruptura da membrana de algumas células da área irradiada. MACHET \& BOUCAUD (2002) resumem o assunto dizendo que a propagação de uma onda ultra-sônica dentro da pele tem duas principais conseqüências físicas: a cavitação e o aquecimento, mecanismos esses que podem estar conectados já que a cavitação pode causar um aquecimento considerável. Tal fato corrobora com o apresentado por JESUS et al, 2006, onde relatam que quando o UST é aplicado por seus efeitos térmicos, os efeitos não térmicos também acontecem.

Em relação aos ativos lipolíticos, nossos dados vêm a confirmar os efeitos positivos na redução da prega cutânea no grupo US+Ativos. Segundo GUYTON, (1997), WAJCHENBERG (2000) e FONSECAALANIZ et. al, (2006), as metilxantinas, além de serem bloqueadoras de $\alpha 1$, também bloqueiam a fosfodiesterase. Dessa forma, o AMPc se mantém ativo, favorecendo a queima de gordura. Quanto à ioimbina, sua ação envolve o bloqueio de $\alpha 2$, o que contribui para a ativação da adenilatociclase e, como conseqüência, também para a queima de gordura. De acordo com MILANI et al (2006) a cafeína e a aminofilina são muito usadas como princípio ativo, por serem estimuladores beta-adrenérgicos e aumentarem a lipólise. A Sociedade Brasileira de Endocrinologia e Metabolismo (2006) afirma que a maior perda de peso ocorre quando a aminofilina é associada à efedrina e à cafeína. Os percentuais desses ativos utilizados no presente estudo corroboram com o que costuma ser utilizado nos mais diversos estudos. Segundo LAMBERT (1982), BELILOWSKI (1988) e BASSOLI (2001), com o objetivo de evitar efeitos os indesejáveis da cafeína, propõem a aplicação tópica de cafeína a 5\%. A ioimbina e aminofilina tem sido utilizada com um percentual máximo de 2\% (RASCOVISKI et. al, 2000).

Em estudo realizado por POLACOW et. al, (2004), foi possível comprovar a permeação do fármaco Tiratricol através da fonoforese, verificando por meio de análise histológica que o UST só foi capaz de promover lise adipocitária quando associado á fonoforese.

No entanto, o fato de que o UST somente seja capaz de promover lise adipocitária quando associando à fonoforese, conforme defendido por alguns autores acaba por confrontar com os resultados apresentados no presente estudo, para o grupo US, quando comparadas as variações percentuais entre os grupos. O grupo US, no que se refere à adipometria e perimetria, apresentou maior variação quando comparado ao CTRL. De acordo com estudos mostrados por BORGES (2006), no que se refere à atuação das ondas ultrassônicas no tecido adiposo, deve-se considerar o fato de que na gordura o coeficiente de absorção é baixo, decorrente da homogeneidade do tecido, absorvendo pouca energia ultrassônica, sendo pouco provável a sua ação lipolítica. Ainda pontua que a energia do UST diminui com a distância do transdutor, e conseqüentemente a dose de US ajustada no aparelho nunca será a mesma que chegará até a área alvo, mesmo no UST de $3 \mathrm{MHz}$, onde os efeitos ocorrem mais superficialmente. Conforme mencionado anteriormente, tais hipóteses confrontam com os resultados desse estudo, assim como podem confrontar com as evidências clínicas do nosso dia-a-dia.

É fato que ocorram efeitos de absorção e atenuação das ondas ultrassônicas, dentre outros, e por isso a dose ajustada não chegue à mesma no tecido alvo. No entanto, buscou-se nesse estudo tentar controlar ao máximo esses efeitos através de algumas medidas: limpeza da área com álcool a 70\% e algodão, a fim de diminuir a impedância tecidual e com isso minimizar fenômeno de reflexão (GUIRRO e GUIRRO, 2004); utilização de substância acopladora manipulada em farmácia de manipulação especializada, em formulação gel, tanto o gel comum quanto o gel com ativos, pois como o gel tem impedância acústica próxima à da pele 
o fenômeno de reflexão também seria minimizado (BORGES, 2006); mensuração da espessura da camada adiposa (subcutânea) por ultrassonografia diagnóstica, o que nos permitiu chegar o mais próximo do valor real e não especular profundidade e espessura do tecido; para a mensuração da intensidade terapêutica considerou-se a dose ideal que deveria chegar ao tecido adiposo em estudo, levando-se em consideração a atenuação das ondas sonoras nos tecidos superficiais ao tecido alvo (pele e tecido subcutâneo). De acordo com Borges, 2006 e Agne, 2005, recomenda-se doses abaixo de $1 \mathrm{~W} / \mathrm{cm}^{2}$ utilizando o ultrassom na forma contínua. Como não se tratava de lesão tecidual consideramos o estado tecidual em fase crônica e a intensidade requerida de $0,8 \mathrm{~W} / \mathrm{cm}^{2}$. Relacionando a intensidade requerida de $0,8 \mathrm{~W} / \mathrm{cm}^{2} \mathrm{com}$ a espessura do tecido, dado através do ultrassom diagnóstico, chegou-se a intensidade a ser utilizada em cada voluntária, a qual variou de $1,12 \mathrm{~W} / \mathrm{cm}^{2}$ a $2 \mathrm{~W} / \mathrm{cm}^{2}$.

Cabe ainda atentar para outro fato relacionado à intensidade do UST. Conforme defendido por BORGES (2006) o UST influência muito pouco o tecido adiposo, quando utilizado em doses baixas, terapêuticas. Entretanto, no procedimento de hidrolipoclasia ultra-sônica, onde o UST é aplicado na região alvo após ter havido a infiltração de soro fisiológico e/ou anestésicos no tecido adiposo, em intensidades bem altas (cerca de 2 a $3 \mathrm{~W} / \mathrm{cm}^{2}$ ), há uma influência significativa das ondas sonoras, provocando cavitação instável na membrana do adipócito e com isso lise adipocitária. O UST também tem sido muito utilizado antes de lipoaspiração, para facilitar a extração da gordura. Alguns pesquisadores puderam verificar no tecido adiposo sonado extraído, grande dano produzido pela cavitação instável (BORGES, 2006). No presente estudo, utilizamos doses acima de $1,12 \mathrm{~W} / \mathrm{cm}^{2}$, com o máximo de $2 \mathrm{~W} / \mathrm{cm}^{2}$ (permitido pelo aparelho), consideradas altas e capazes de gerar efeito de cavitação instável em adipócitos. Dentre esse e outros fatos acima mencionados acreditamos, talvez, serem os responsáveis por proporcionar esses resultados positivos no grupo US.

Nos resultados desse estudo, no que se refere à perimetria, o grupo US+Ativos não apresentou diferença significativa na variação percentual entre os grupos quando comparado ao CTRL. Segundo MARINS \& GIANNICHI (2003) essa técnica de avaliação, apesar de ser extremamente simples, requer alguns cuidados, para evitar resultados distorcidos; dentre eles a colocação da fita em ponto previamente demarcado, com precisão, através de um lápis demográfico, sem exercer pressão excessiva, nem deixar a fita solta, assim como visualizar a uniformidade do alinhamento da fita. Dessa forma, mesmo sendo a avaliação realizada por um mesmo avaliador durante todo o experimento, postulamos que alguns desses cuidados possam ter sido omitidos nesse grupo, considerando-se o fato de que outro método de avaliação, a adipometria, demonstrou resultado positivo para as mesmas pacientes, no mesmo espaço de tempo e em decorrência dos mesmos tratamentos.

A avaliação dos grupos estudados, realizada pela Ultrassonografia Diagnóstica, demonstrou que após o tratamento somente o grupo US obteve redução significativa da espessura da camada adiposa, em relação ao pré. Tal resultado demonstra que houve redução da espessura da camada adiposa no grupo US pós tratamento, quando comparado ao pré-tratamento. Comparando-se as variações percentuais entre os grupos, o grupo US foi o que apresentou a maior variação quando comparado ao CTRL.

Em estudo realizado por DINIZ et. al, (2009), a ultrassonografia mostrou ser um exame altamente reprodutível e capaz de aferir as espessuras das gorduras subcutânea e visceral. Nesse estudo, a tomografia computadorizada de abdome foi considerada método "padrão-ouro" para a determinação da gordura abdominal em virtude da sua capacidade de diferenciar a adiposidade subcutânea e visceral, além de ser um método altamente reprodutível. Um fator que favorece essa pela tomografia computadorizada e pela ressonância magnética é o fato de estes métodos não dependerem da habilidade do operador para identificar as estruturas durante o exame, assim como não sofrerem influência da pressão do transdutor sobre o abdome durante a obtenção das medidas (DINIZ et. al, 2009). Entretanto, a radiação presente na tomografia computadorizada e o alto custo e o tempo de execução da ressonância magnética são aspectos desfavoráveis para utilizá-los na rotina para quantificação da gordura abdominal (RADOMINSKI et. al, 2000; DINIZ et. al, 2009).

Ribeiro Filho (2006), diz que apesar da ultrassonografia requerer equipamento e pessoal especializados à semelhança de outros, é significativamente menos dispendioso que a tomografia computadorizada, tratando-se de um método inócuo, de fácil e rápida execução, com boa especificidade e reprodutibilidade, principalmente para estudo da adiposidade visceral.

Nos resultados da variável ultrassonografia o grupo US+Ativos não apresentou diferença significativa na variação percentual entre os grupos quando comparado ao CTRL. Segundo DINIZ et. al, (2009), algumas limitações técnicas no procedimento de ultrassonografia devem ser citadas, uma vez que elas podem dificultar a execução do exame e limitar sua confiabilidade. Entre elas se observa a não 
cooperação do paciente em manobras de respiração, transdutores inadequados e especialmente o treinamento e técnica inadequados do operador. Dessa forma, considerando o fato do método diagnóstico por ultrassonografia, segundo alguns autores, não ser "padrão-ouro" para avaliação da gordura subcutânea, como também o fato de que, mesmo sendo a avaliação realizada por um mesmo avaliador durante todo o experimento, médico radiologista especializado, postulamos que alguns desses cuidados possam ter sido também omitidos nesse grupo. Tal suposição se faz presente nesse estudo uma vez que outro método de avaliação, a adipometria, demonstrou resultado positivo para as mesmas pacientes, no mesmo espaço de tempo e em decorrência dos mesmos tratamentos; assim como também demonstrou resultados positivos no grupo US.

No que se refere aos resultados do grupo CTRL foi possível verificar que as voluntárias apresentaram redução na adipometria e perimetria. Para a Sociedade Brasileira de Endocrinologia e Metabolismo (2006), perdas de peso podem resultar em redução de medidas corporais. Como não se constituiu foco desse trabalho avaliar ou controlar a dieta das participantes, postulamos que a perda de medidas exibida pelas integrantes do grupo CTRL possa ter sido resultado de alguma variação em suas dietas.

\section{CONCLUSÃO}

O presente estudo evidenciou dados favoráveis que justificam a utilização do ultrassom terapêutico de $3 \mathrm{MHz}$, no modo contínuo, associado à ativos lipolíticos, como as metilxantinas e a ioimbina, na redução da adiposidade infra-abdominal. Da mesma forma, dados referentes à utilização do ultrassom terapêutico na forma isolada também foram significativamente favoráveis, e têm a sua utilização fundamentada nesse estudo.

No que se refere aos métodos de avaliação, a adipometria mostrou ser nesse estudo um método mais eficaz e de maior credibilidade para a mensuração do tecido adiposo subcutâneo, apresentando menor limitação técnica, quando comparado à perimetria e à ultrassonografia.

Faz-se necessária a realização de novas pesquisas, com dieta controlada, comparando o ultrassom terapêutico de $3 \mathrm{MHz}$ nos modos contínuo e pulsado, assim como nas formas isolada e associada aos ativos lipotíticos, contribuindo dessa forma para o enriquecimento e credibilidade dessa terapêutica na Fisioterapia Dermatofuncional.

\section{REFERÊNCIAS BIBLIOGRÁFICAS}

AGNE, J. E. Eletrotermoterapia: Teoria e Prática. Santa Maria: Orium, 2005.

BASSOLI, D. A. Avaliação dos efeitos do ultra-som pulsado de baixa intensidade na regeneração de músculos esqueléticos com vistas à aplicabilidade em clínica fisioterapêutica. Dissertação (Mestrado). Escola de Engenharia de São Carlos / Faculdade de Medicina de Ribeirão Preto / Instituto de Química de São Paulo, Universidade de São Paulo, São Paulo, 2001.

BELILOWSKY, M. Cellulite et caféine precutanée: études récents sur percutaféine. Compres Rendus de Thérapeutique et de Pharmacologic Clinique. v. 6, n. 60, p. 23-27, 1988..

BORGES, F. S. Dermatofuncional: modalidades terapêuticas nas disfunções estéticas. São Paulo: Phorte, 2006.

CAGNIE, B.; ELKE VINCK; STEVEN RIMBAUT; GUY VANDERSTRAETEN. Phonophoresis versus topical application of ketoprofen: comparison between tissue and plasma levels. Phys Ther. v. 83, n. 70, p. 7-12, 2003. .

CAMPOS, M. S. P. Curso de fisioterapia estética corporal. set. 2000. 
DINIZ, A. L. D.; TOMÉ, R.A.F.; DEBS,C.L.; CARRARO,R.; ROEVER,L.B.; COSTA PINTO,R.M.; Avaliação da reprodutibilidade ultra-sonográfica como método para medida da gordura abdominal e visceral. Radiol Bras. v. 42, n. 6, p. 353-357, 2009.

ERVALHO, L. A. Os efeitos do ultra-som 3 MHZ em mulheres com fibroedema gelóide grau II na região glútea trocantérica: Relato de caso. Centro Brasileiro de Estudos Sistêmicos. Porto Alegre-RS. 2005, Monografia..

FONSECA- ALANIZ, Miriam H.; TAKADA, Julie; ALONSO- VALE, Maria Isabel C.; LIMA, Fabio Bessa. O tecido adiposo como centro regulador do metabolismo. Arq. Brás. Endocrinol. Metab. v. 50, n. 2, p. 216-229, 2006.

GARCIA, E. A. C. Biofísica. São Paulo: Savier, 2000.

GILMAN, A. G. As Bases Farmacológicas da Terapêutica, 10 ed. Rio de Janeiro: Mcgraw Hill, 2003.

GUIRRO, E.; GUIRRO, R. Fisioterapia dermatofuncional: fundamentos, recursos e patologias. 3 ed. São Paulo: Manole, 2002.

GUIRRO, E.; GUIRRO, R. Fisioterapia dermato-funcional: Fundamentos, recursos e patologias. 3 ed. São Paulo: Manole, 2004.

GUYTON, A. C.; HALL, J. E. Tratado de Fisiologia Médica. 9 ed. Rio de Janeiro, Ed. Elsevier, 1997. .

HERCOX, B.; MEHRETEAB, T.A.; WISBERG, J. Physical Agents: a comprehensive text for physical therapists. Norwalk, Connecticut: Appleton \& Lange, 1994.

JESUS, G. S.; FERREIRA, A. S.; MENDONÇA, A. C. Fonoforese x permeação cutânea. Fisioter Mov. v. 19, n. 4, p. 83-89, 2006.

KOEKE, P. U. Estudo comparativo da eficácia da fonoforese, do ultra-som terapêutico e da aplicação tópica de hidrocortisona no tratamento do tendão de ratos em processo de reparo tecidual. Dissertação (Mestrado). Instituto de Bioengenharia, Universidade de São Paulo, São Paulo, 2003.

LAMBERT, J. Traitemente par la percutafèine dês lipodystrophies localisées. Compts Rendus de Thérapeutiques et de Pharmacologic Clinique. v. 1, n. 10, p. 3-6, 1982.

LOW, J.; REED, A. Ultra-som Terapêutico. Eletroterapia Explicada Princípios e Prática. 3 ed. São Paulo: Manole, 2001.

MACHADO, C.M. O uso do ultra-som na estética. São Paulo: Vida Estética, 2001.

MARINS, J. C. B.; GIANNICHI, R. S. Avaliação e Prescrição de Atividade Física. 3 ed. Rio de Janeiro: Afiliada, 2003.

MILANI, G. B.; JOÃO, S. M. A.; FARAH, E. A. Fundamentos da Fisioterapia Dermato-funcional: revisão de literatura. Fonte: Fisioterapia e Pesquisa, 12(3), 2006.

MORETTI, E. A. Estudo macro e microscópico da fáscia superficial em membros inferiores: um conceito diferente sobre a celulite. Mesoterapia Atual, p. 9-12, 1997.

POLACOW, M. L. O.; PIRES-DE-CAMPOS,M. S. M.; LEONARDI,G.R.; CARVALHO, L.; RIBEIRO,M.C.A.P.; MONTEBELLO,M.I.L. Efeitos do ultra-som na permeação cutânea do Tiratricol: Análise Histológica. Rev Bras Fisioter. v. 8, n. 1, p. 53-60, 2004. 
RASCOVSKI, A.; MILLNER, T.H.; BATALHA, L.; REIS,C.; MANCINI, M.C.; HALPERN,A. Eficácia e tolerabilidade das substâncias calorigênicas: ioimbina, triiodotironina, aminofilina combinada a efedrina e fenilpropanolamina no tratamento da obesidade a curto prazo. Arq Bras Endocrinol Metab. v. 44, n. 1, p. 95$102,2000$.

RIBEIRO FILHO, F. F.; MARIOSA, LIDIA S.; FERREIRA, SANDRA R.G.;ZANELLA, MARIA TERESA Gordura visceral e síndrome metabólica: mais que uma simples associação. Arq Bras Endocrinol Metab. v. 50, n. 2, p. 230-238, 2006.

SANTOS, L; XIMENES, O. A Efetividade do Ultra-Som Terapêutico associado à drenagem Linfática na paciente com Fibro Edema Gelóide. Revista KINESIA. Ed. 3, Ano 2, 1º semestre, mar. 2010.

SCHUH, C. M.; JANK, B.; FORTES, A.P. Uso da Eletrolipoforese associada à Drenagem Linfática Manual na redução de medida corporal na região abdominal. Revista KINESIA. Ed. 3, Ano 2, $1^{\circ}$ semestre, mar. 2010.

Sociedade Brasileira de Endocrinologia e Metabologia. Perda de Peso: Tratamentos Heterodoxos e Suplementos Nutricionais. Projeto Diretrizes da Associação Médica Brasileira e Conselho Federal de Medicina. jun. 2006.

STARKEY, C. Recursos Terapêuticos em Fisioterapia. 2 ed. São Paulo: Manole, 2001.

TOGNI,A.B. Avaliação dos efeitos ultra- som associado à fonoforese e endermologia no tratamento do fibro edema gelóide. Universidade do Sul de Santa Catarina. Tubarão, 2006.

WAJCHENBERG, B. L. Tecido adiposo como glândula endócrina. Arq Bras Endocrinol Metab. v. 44, n. 1, p. 13-20, 2000. 